\title{
IMPACTO DE LOS FACTORES EXTERNOS EN EL PRODUCTO BRUTO INTERNO PERUANO DURANTE 1994-2018
}

\author{
José Franco Iparraguirre ${ }^{A}$ | Fernando Cuyutupac Borja ${ }^{B}$
}

\begin{abstract}
RESUMEN
Perú como economía pequeña y abierta esta propensa a sufrir choques externos que originen fluctuaciones en su PBI, además dado el alto grado de dependencia de las materias primas esto podría amplificarse. Con relación a esto, el presente trabajo pretende cuantificar a través de un modelo de vectores autorregresivos con la incorporación de variables exógenas, cuan vulnerable es la economía peruana a choques externos y cómo afectaría al crecimiento económico una agudización de las fricciones comerciales actuales.
\end{abstract}

JEL: E01; C22; F63

PALABRAS CLAVES: Perú; choques externos; modelo VARX; crecimiento económico.

\section{ABSTRACT}

Peru as a small and open economy is prone to external shocks that cause fluctuations in its GDP, in addition to the high degree of dependence on raw materials this could be amplified. In this regard, the present work aims to quantify through a model of autoregressive vectors with the incorporation of exogenous variables, how vulnerable the Peruvian economy is to external shocks and how an intensification of current commercial frictions would affect economic growth.

JEL: E01; C22; F63

KEYWORDS: Peru; External Shocks; VARX Model; Economic Growth

\footnotetext{
A Investigador Docente de la Escuela Profesional de Economía de la Facultad de Ciencias Contables, Económicas y Financieras de la Universidad de San Martín de Porres.

B Economista de la Escuela Profesional de Economía de la Facultad de Ciencias Contables, Económicas y Financieras de la Universidad de San Martín de Porres.
} 


\section{INTRODUCCIÓN}

Esta investigación pretende analizar la relevancia de los factores externos en el crecimiento del producto bruto interno durante el período de 1994 - 2018, esto con el propósito de saber hasta qué punto el crecimiento económico ha sido impulsado por factores externos con respecto a los domésticos, y cuan vulnerable es la economía peruana ante cambios en las condiciones externas.

La literatura existente sobre ciclos económicos $\mathrm{y}$ vulnerabilidad externa es abundante $\mathrm{y}$ ha tenido mayor notoriedad en los últimos años, las principales investigaciones han abordado este tema desde diversas aristas, analizando la implicancia de los factores externos sobre la balanza de pagos, reservas internacionales, crecimiento en el empleo, tipo de cambio real y el crecimiento económico.

Dado que Perú es una economía dependiente de los productos básicos entre los mercados emergentes, característica que ha venido siendo más notoria en los últimos años, muestra de ellos es el alto nivel de representación de estos productos sobre el total de exportaciones, según estadística oficial del Banco Central de Reserva del Perú (BCRP) representan más del 50\% del total de exportaciones totales para el período de análisis. Ante esta situación, una correcta especificación del grado de vulnerabilidad ante posibles cambios en el contexto internacional servirá como referente para modificar las políticas actuales e intentar corregir las falencias actuales a fin de tener una economía más diversificada. Es por ello que el objetivo de esta investigación es determinar el impacto proveniente de los factores externos que generan fluctuaciones en la economía y a su vez cuantificar el grado de vulnerabilidad de la economía peruana frente a cambios provenientes de los mismos.

La segunda parte de este trabajo aborda los antecedentes y marco teórico. En la tercera parte se muestran los datos y la metodología empleada. En la cuarta y quinta parte se muestran los resultados $\mathrm{y}$ conclusiones $\mathrm{y}$ recomendaciones respectivamente.

\section{ANTECEDENTES Y MARCO TEÓRICO}

Importancia y consecuencia de la irrupción de capitales en la economia

Conforme transcurrían los primeros años de la década de los 1990, los flujos de capital extranjero comenzaron a regresar a los países latinoamericanos después de la sequía que siguió a la crisis de la deuda de la década de 1980 . En la mayoría de los países, esta renovación de las entradas de capital se asoció con mercados en auge de activos, apreciación del tipo de cambio real, inversiones en auge y un boyante crecimiento económico.

A raíz de toda esta euforia, (Calvo, Leiderman, \& Reinhart, 1993) observaron con sorpresa que, aunque la región estaba en un proceso de reforma sustancial, ingentes cantidades de capital fluían hacia la mayoría de los países latinoamericanos. Es preciso aclarar que este fenómeno se dio a pesar de las grandes diferencias existentes en cuestiones de políticas macroeconómicas y desempeño económico entre cada uno de ellos. Sugirieron que los factores externos, un choque común en toda la región, desempeñaron un papel importante en todo este fenómeno. En ese momento, argumentaron que la caída de las tasas de interés en los Estados Unidos, una continua recesión y la evolución de la balanza de pagos en dicho país, alentaba a los inversores a buscar mejores oportunidades de inversión en el extranjero.

Resultado de estos hallazgos, conllevaron a una preocupación, y esta era que los factores externos podrían deteriorarse tan fácilmente como habían mejorado durante la época de bonanza, con consecuencias potencialmente graves para la región. La crisis del Tequila en 1995, y lo más importante para América Latina, la crisis rusa de 1998 y el consiguiente colapso de las entradas de capital a la región, con graves consecuencias económicas y financieras, fueron estudiadas por (Calvo \& Talvi, 2005) y respaldaron las preocupaciones premonitorias planteadas por (Calvo, Leiderman, \& Reinhart, 1993). La sequía de entradas de capital a la región después de la crisis rusa duró hasta finales de 2002.

Muchos años después, la aparición de Asia y, en particular, de China como actor global ha cambiado drásticamente el panorama de los mercados de productos básicos y financieros, en este último caso a través de la exportación de ahorros financieros, en el primero a través de un fuerte aumento en la demanda de productos primarios. No es sorprendente que economías como la peruana hayan experimentado desde entonces una nueva fase de precios en auge de los activos, que aprecian los tipos de cambio reales, las inversiones que presentan un crecimiento boyante y un sólido crecimiento económico. 
Los grandes movimientos de términos de intercambio pueden tener importantes implicaciones para el desempeño macroeconómico a medida que cambian los precios relativos y los ingresos. El auge de los productos básicos ha llegado a su fin debido a que los precios de los mismos han ido disminuyendo constantemente desde 2011, y es probable que este shock externo sea persistente. Los precios de los productos básicos experimentaron un notable aumento durante la década de 2000, el llamado superciclo de los productos básicos, que se vio ligeramente interrumpido por la crisis financiera mundial, lo que generó un auge en los términos de intercambio (TI) para muchas economías exportadoras de productos básicos. Por ejemplo, en Chile y Perú, los términos de intercambio se duplicaron de 2000 a 2011, y en Colombia aumentaron en un 70 por ciento (Roch, 2017). Sin embargo, los precios de los metales y el petróleo disminuyeron después de 2011 y mediados de 2014, respectivamente. Debido al deterioro de la TI, el impacto resultó en menores ingresos nacionales, déficits de cuenta corriente más amplios y monedas nacionales más débiles.

Sin embargo, un marco político de alta calidad mitiga esta vulnerabilidad. La combinación de un marco de política fiscal sólido, un régimen creíble de metas de inflación y un régimen de tipo de cambio flexible aumenta la resistencia de Perú a los cambios en las condiciones externas.

El grado de apertura comercial de Perú es significativo, ya que fue testigo de un proceso de mayor integración comercial con el resto de las economías en las últimas dos décadas, respaldado por reducciones arancelarias unilaterales de importación y la firma de varios acuerdos de libre comercio, incluso con los Estados Unidos y la Unión Europea. De hecho, la economía peruana muestra uno de los mayores niveles de apertura comercial de la región.

Hoy en día, Perú, es una de las economías de mercados emergentes con la integración financiera internacional más profunda, medida como la suma de los activos y pasivos extranjeros totales, en relación con el PIB. Se obtiene una imagen similar utilizando una medida de apertura de la cuenta de capital basada en el índice construido por (Chinn \& Ito, 2006)

En palabras de (Castillo, Montoro, \& Tuesta, 2007) la economía peruana puede ser caracterizada como una economía pequeña y abierta. Y por tal motivo, es imperativo estudiar las fluctuaciones económicas y los potenciales movimientos estructurales desde un punto de vista internacional, esto con el objetivo de comprender como los shocks externos de cantidades y precios influyen sobre la performance de las variables macroeconómicas más relevantes.

Notable es la integración financiera internacional y apertura comercial de Perú; las materias primas, en particular, representan una parte importante de las exportaciones, por lo que la economía es potencialmente vulnerable a los cambios en las condiciones financieras mundiales, la demanda externa y los precios de los productos básicos

\section{Marco teórico}

\begin{tabular}{|c|c|c|c|c|c|c|}
\hline Autores & Título & Período & Variables & Metodología & Objetivos & Hallazgos \\
\hline $\begin{array}{l}\text { (Izquierdo, } \\
\text { Romero- } \\
\text { Aguilar, \& } \\
\text { Talvi, 2008) }\end{array}$ & $\begin{array}{l}\text { Booms and } \\
\text { busts in Latin } \\
\text { America: The } \\
\text { role of external } \\
\text { factors }\end{array}$ & $\begin{array}{c}1990 \mathrm{q} 1 \\
- \\
2006 \mathrm{q} 4\end{array}$ & $\begin{array}{l}\text {-PBI per cápita } \\
\text { (ALC7). } \\
\text {-Índice de } \\
\text { producción } \\
\text { industrial. } \\
\text {-Índice de } \\
\text { términos de } \\
\text { intercambio } \\
\text { regionales. } \\
\text {-Rendimiento } \\
\text { de los bonos del } \\
\text { tesoro (EE. UU). } \\
\text {-Diferencial sobre } \\
\text { los bonos de alto } \\
\text { rendimiento sobre } \\
\text { los bonos del } \\
\text { tesoro. }\end{array}$ & $\begin{array}{l}\text { Modelo de } \\
\text { corrección } \\
\text { de errores } \\
\text { vectoriales }\end{array}$ & $\begin{array}{l}\text {-Evaluar la influencia } \\
\text { de los factores } \\
\text { externos en los } \\
\text { auges y crisis } \\
\text { experimentados } \\
\text { en América } \\
\text { Latina (analizaron } \\
\text { el crecimiento } \\
\text { económico de las } 7 \\
\text { principales economías } \\
\text { latinoamericanas) }\end{array}$ & $\begin{array}{l}\text { - Las diferencias } \\
\text { dinámicas de los } \\
\text { factores externos } \\
\text { pueden explicar } \\
\text { significativas } \\
\text { diferencias en } \\
\text { el desempeño } \\
\text { del crecimiento } \\
\text { económico. } \\
\text {-Una reversión en } \\
\text { las condiciones } \\
\text { financieras externas } \\
\text { de una magnitud } \\
\text { observada en el pasado } \\
\text { tendrá un gran impacto } \\
\text { en el PBI de América } \\
\text { Latina. }\end{array}$ \\
\hline
\end{tabular}




\begin{tabular}{|c|c|c|c|c|c|c|}
\hline $\begin{array}{c}\text { (Abrego \& } \\
\text { Österholm, } \\
\text { 2010) }\end{array}$ & $\begin{array}{l}\text { External } \\
\text { linkages and } \\
\text { economic } \\
\text { growth in } \\
\text { Colombia: } \\
\text { Insights from a } \\
\text { Bayesian VAR } \\
\text { model }\end{array}$ & $\begin{array}{c}1995 \mathrm{q} 2 \\
- \\
2007 \mathrm{q} 2\end{array}$ & $\begin{array}{l}\text {-Log del PBI real } \\
\text { mundial. } \\
\text {-Tasa de los } \\
\text { T-Bonds } \\
\text {-EMBI. } \\
\text {-Bonos } \\
\text { corporativos de } \\
\text { alto rendimiento. } \\
\text {-IED. } \\
\text {-Log PBI real de } \\
\text { Colombia. } \\
\text {-Log gasto } \\
\text { del gobierno } \\
\text { colombiano. } \\
\text {-Tasa nominal } \\
\text { del crédito en } \\
\text { Colombia. }\end{array}$ & VAR Bayesiano & $\begin{array}{l}\text {-Determinar hasta qué } \\
\text { punto el crecimiento } \\
\text { económico ha sido } \\
\text { impulsado por factores } \\
\text { externos. } \\
\text {-Medir la sensibilidad } \\
\text { del crecimiento } \\
\text { a cambios en las } \\
\text { condiciones externas. }\end{array}$ & $\begin{array}{l}\text {-El efecto del } \\
\text { crecimiento global } \\
\text { tiene un impacto } \\
\text { considerable en el PBI } \\
\text { colombiano. } \\
\text { - Una desaceleración } \\
\text { leve del PBI global } \\
\text { se trasladaría en una } \\
\text { caída considerable en } \\
\text { el PBI de Colombia, } \\
\text { pero con una rápida } \\
\text { recuperación. }\end{array}$ \\
\hline $\begin{array}{c}\text { (Adler \& } \\
\text { Sosa, 2011) }\end{array}$ & $\begin{array}{l}\text { Commodity } \\
\text { price cycles: } \\
\text { The perils of } \\
\text { mismanaging } \\
\text { the boom }\end{array}$ & $\begin{array}{l}1970- \\
2010\end{array}$ & $\begin{array}{l}\text {-Crecimiento del } \\
\text { PBI. } \\
\text {-Términos de } \\
\text { intercambio. } \\
\text {-Posición Externa. } \\
\text {-Fundamentos } \\
\text { propios del país. } \\
\text {-Condiciones } \\
\text { externas }\end{array}$ & $\begin{array}{l}\text { - Enfoque } \\
\text { de corte } \\
\text { transversal }\end{array}$ & $\begin{array}{l}\text { Examinar el } \\
\text { comportamiento } \\
\text { de los precios y las } \\
\text { tendencias en la } \\
\text { dependencia de los } \\
\text { productos básicos y la } \\
\text { concentración de las } \\
\text { exportaciones en las } \\
\text { principales regiones de } \\
\text { mercados emergentes } \\
\text { durante } 1970 \text { - } 2010 \text {. }\end{array}$ & $\begin{array}{l}\text {-Sudamérica es } \\
\text { más dependiente de } \\
\text { productos básicos y } \\
\text { esto se ha hecho más } \\
\text { evidente en los últimos } \\
\text { años, mientras Asia } \\
\text { emergente pasó a ser } \\
\text { un importador neto. } \\
\text {-Los exportadores de } \\
\text { metales (Chile y Perú) } \\
\text { aún presentan una } \\
\text { concentración alta en } \\
\text { sus exportaciones. }\end{array}$ \\
\hline (Roch, 2017) & $\begin{array}{l}\text { The adjustment } \\
\text { to commodity } \\
\text { price shocks } \\
\text { in Chile, } \\
\text { Colombia, and } \\
\text { Peru }\end{array}$ & $\begin{array}{l}1999 \mathrm{q} 1- \\
2015 \mathrm{q} 4\end{array}$ & $\begin{array}{l}\text {-Índice de precios } \\
\text { de productos } \\
\text { básicos. } \\
\text {-Gasto público. } \\
\text {-ingreso público. } \\
\text {-PBI real. } \\
\text {-Tipo de cambio } \\
\text { real. } \\
\text {-Cuenta corriente. }\end{array}$ & $\begin{array}{c}\text { VAR } \\
\text { Multivariado }\end{array}$ & $\begin{array}{l}\text { Realizar un análisis } \\
\text { comparativo del } \\
\text { ajuste a nivel macro } \\
\text { en Chile, Colombia } \\
\text { y Perú a los choques } \\
\text { de intercambio de } \\
\text { productos básicos. }\end{array}$ & $\begin{array}{l}\text {-A pesar de la } \\
\text { desaceleración global, } \\
\text { el crecimiento del PBI } \\
\text { se mantuvo resistente } \\
\text { a los shocks, esto } \\
\text { evidencia solidez en } \\
\text { sus políticas. }\end{array}$ \\
\hline $\begin{array}{l}\text { (Corbo \& } \\
\text { Schmidt- } \\
\text { Hebbel, } \\
\text { 2011) }\end{array}$ & $\begin{array}{l}\text { The } \\
\text { international } \\
\text { crisis and } \\
\text { Latin America: } \\
\text { Growth } \\
\text { effects and } \\
\text { development } \\
\text { strategies }\end{array}$ & $\begin{array}{c}1990 \mathrm{q} 1 \\
- \\
2009 \mathrm{q} 4\end{array}$ & $\begin{array}{l}\text {-Amplitud de la } \\
\text { caída del PBI (\%). } \\
\text {-Variables de LP. } \\
\text {-Variables } \\
\text { estructurales. } \\
\text {-Variables cíclicas } \\
\text { externas. } \\
\text {-Variables de PI } \\
\text {-Interacciones }\end{array}$ & $\begin{array}{c}\text { Regresiones } \\
\text { con Datos Panel }\end{array}$ & $\begin{array}{l}\text {-Analizar la manera } \\
\text { en que las condiciones } \\
\text { estructurales de } \\
\text { A.L. han afectado } \\
\text { su vulnerabilidad a } \\
\text { choques externos. } \\
\text {-Evaluar el rol de los } \\
\text { distintos mecanismos } \\
\text { de transmisión. }\end{array}$ & $\begin{array}{l}\text {-Persistencia de } \\
\text { una considerable } \\
\text { heterogeneidad en los } \\
\text { regímenes y políticas } \\
\text { de los países de AL. } \\
\text { - La adopción } \\
\text { de estrategias de } \\
\text { desarrollo ha llevado } \\
\text { a que AL tenga una } \\
\text { notable resistencia a } \\
\text { los choques externos } \\
\text { negativos. }\end{array}$ \\
\hline
\end{tabular}




\begin{tabular}{|c|c|c|c|c|c|c|}
\hline (Tello, 2012) & $\begin{array}{l}\text { Peru: } \\
\text { integration, } \\
\text { sectoral } \\
\text { specialization } \\
\text { and } \\
\text { synchronization } \\
\text { with } \\
\text { international } \\
\text { gross domestic } \\
\text { product cycles }\end{array}$ & $\begin{array}{l}1982- \\
2006\end{array}$ & $\begin{array}{l}\text {-PBI Perú. } \\
\text {-Grado de } \\
\text { especialización } \\
\text { sectorial. } \\
\text {-Grado Integración } \\
\text { Financiera. } \\
\text {-Grado Integración } \\
\text { comercial. } \\
\text {-Grado de } \\
\text { sincronización. }\end{array}$ & $\begin{array}{c}\text { Estimación } \\
\text { de ecuaciones } \\
\text { simultáneas con } \\
\text { panel data. }\end{array}$ & $\begin{array}{l}\text {-Analizar las } \\
\text { interrelaciones } \\
\text { entre el grado de } \\
\text { sincronización del } \\
\text { ciclo del PBI peruano } \\
\text { con los ciclos del PBI } \\
\text { de sus socios. }\end{array}$ & $\begin{array}{l}\text {-Un alto nivel de } \\
\text { integración comercial } \\
\text { en Perú profundizaría } \\
\text { los efectos del ciclo } \\
\text { del PBI de los países } \\
\text { socios en el PBI } \\
\text { peruano. } \\
\text {-Las políticas } \\
\text { económicas enfocadas } \\
\text { en diversificar la } \\
\text { canasta de exportación } \\
\text { y el destino de estos, } \\
\text { atenuaría los efectos } \\
\text { negativos de las crisis } \\
\text { internacionales. }\end{array}$ \\
\hline $\begin{array}{c}\text { (Saldarriaga } \\
\& \\
\text { Winkelried, } \\
2013 \text { ) }\end{array}$ & $\begin{array}{l}\text { Trade linkages } \\
\text { and growth in } \\
\text { Latin America: } \\
\text { An SVAR } \\
\text { analysis. }\end{array}$ & $\begin{array}{c}1989 \mathrm{q} 1 \\
-2011 \mathrm{q} 2\end{array}$ & $\begin{array}{l}\text {-Tasas de } \\
\text { crecimiento de } 29 \\
\text { países alrededor } \\
\text { del mundo. } \\
\text {-Participación en } \\
\text { las exportaciones } \\
\text { de AL y países } \\
\text { seleccionados. }\end{array}$ & $\begin{array}{c}\text { VAR } \\
\text { Estructural } \\
\text { (SVAR) }\end{array}$ & $\begin{array}{l}\text {-Analizar los ciclos } \\
\text { económicos existentes } \\
\text { entre América Latina } \\
\text { y China. } \\
\text {-Estudiar la } \\
\text { reasignación del } \\
\text { PBI y la demanda } \\
\text { internacional a ante } \\
\text { cambios en los } \\
\text { patrones de comercio } \\
\text { internacional. }\end{array}$ & $\begin{array}{l}\text { Las variaciones en los } \\
\text { patrones de comercio } \\
\text { internacional reflejan } \\
\text { un grado más alto de } \\
\text { sincronización de los } \\
\text { ciclos económicos } \\
\text { entre las economías } \\
\text { emergentes. } \\
\text {-Los ciclos } \\
\text { económicos entre } \\
\text { América Latina y } \\
\text { China se han vuelto } \\
\text { más correlacionados. }\end{array}$ \\
\hline $\begin{array}{l}\text { (Zettelmeyer } \\
\text { \& Österholm, } \\
\text { 2007) }\end{array}$ & $\begin{array}{l}\text { The Effect } \\
\text { of External } \\
\text { Conditionson } \\
\text { Growth in Latin } \\
\text { America }\end{array}$ & $\begin{array}{c}1994 \mathrm{q} 1 \\
- \\
2006 \mathrm{q} 4\end{array}$ & $\begin{array}{l}\text {-Crecimiento PBI } \\
\text { mundial. } \\
\text {-Crecimiento de } \\
\text { AL6 } \\
\text {-Índice ponderado } \\
\text { de los productos } \\
\text { básicos. } \\
\text {-T-bonds (EE. } \\
\text { UU). } \\
\text {-EMBI } \\
\text {-Bonos de alto } \\
\text { rendimiento en } \\
\text { EE. UU. }\end{array}$ & $\begin{array}{l}\text { BVAR con prior } \\
\text { informativos } \\
\text { de estado } \\
\text { estacionario }\end{array}$ & $\begin{array}{l}\text { Analizar la } \\
\text { sensibilidad del } \\
\text { crecimiento del PBI } \\
\text { latinoamericano a } \\
\text { los shocks externos, } \\
\text { tanto reales como } \\
\text { financieros. }\end{array}$ & $\begin{array}{l}\text { Entre un } 50 \text { a } 60 \% \\
\text { de la variación del } \\
\text { PBI latinoamericano } \\
\text { es explicado por los } \\
\text { choques externos. } \\
\text { Los fundamentos de } \\
\text { AL son consistentes } \\
\text { para poder } \\
\text { contrarrestar la caída } \\
\text { de los precios de los } \\
\text { commodities y caídas } \\
\text { en el PBI mundial. }\end{array}$ \\
\hline (Sosa, 2012) & $\begin{array}{c}\text { Output } \\
\text { Fluctuations } \\
\text { in Chile-The } \\
\text { Role of External } \\
\text { Factors }\end{array}$ & $\begin{array}{c}1990 \mathrm{q} 1 \\
-2011 \mathrm{q} 4\end{array}$ & $\begin{array}{c}\text {-PBI real chileno } \\
\text {-VIX } \\
\text {-Promedio } \\
\text { ponderado del } \\
\text { PBI real del G7 y } \\
\text { China. } \\
\text {-Precio } \\
\text { internacional del } \\
\text { cobre (términos } \\
\text { reales) }\end{array}$ & $\begin{array}{c}\text { Vectores } \\
\text { Autorregresivos } \\
\text { Estándar }\end{array}$ & $\begin{array}{l}\text {-Cuantificar el } \\
\text { impacto potencial de } \\
\text { las perturbaciones } \\
\text { externas en la } \\
\text { actividad económica } \\
\text { de Chile. } \\
\text {-Determinar cómo } \\
\text { afectarán a Chile los } \\
\text { nuevos episodios de } \\
\text { aversión al riesgo } \\
\text { global o una fuerte } \\
\text { caída en los precios } \\
\text { del cobre. }\end{array}$ & $\begin{array}{l}\text {-Choques externos } \\
\text { (reales y financieros), } \\
\text { tienen un impacto } \\
\text { significativo en el PBI } \\
\text { chileno, ocasionando } \\
\text { fluctuaciones en el } \\
\text { mismo. } \\
\text {-Un marco de políticas } \\
\text { sólido dará soporte a } \\
\text { la economía de Chile } \\
\text { para hacer frente } \\
\text { su exposición a los } \\
\text { choques externos. }\end{array}$ \\
\hline
\end{tabular}

\section{DATOS Y METODOLOGÍA}

(Stock \& Watson, 2001), señalan que los modelos VAR son útiles para realizar tareas habituales de los macroeconometristas, tales como descripción y resumen de los datos macroeconómicos, pronósticos, cuantificar respuestas a shocks $\mathrm{y}$ elegir el mejor modelo teórico y hacer análisis y recomendaciones de política. Antes de ello (Sims, 1980), pone de manifiesto que, si se pueden usar modelos de pronóstico para el análisis de políticas, y que cualquier modelo de toma de decisiones debe incorporar algunas suposiciones 
de identificación que le permitan pronosticar los efectos de los escenarios alternativos. Argumenta también que los modelos de vectores autorregresivos tienen la ventaja de permitir que se mida la incertidumbre. (Lütkepohl, 2007), por otro lado, menciona que el VAR a estimar debe incluir variables apropiadas de acuerdo con la teoría económica a fin de que el modelo debe ser parsimonioso.

El vector incluye un bloque de factores externos (demanda global, términos de intercambio, $\mathrm{y}$ condiciones financieras internacionales) y también el PBI peruano, (ver gráfica 1). La demanda global está representada por el PBI de China. Los términos de intercambio que se emplea en lugar de un índice de precio de los metales, ya que se pretende capturar también posibles efectos positivos en las fluctuaciones, las condiciones externas están determinadas por el índice de volatilidad financiera, que según (Bloom, 2009), tienen un alto grado de correlación con la incertidumbre tanto a nivel macro como micro, además se optó por incluir este índice dado que arroja resultados muy parecidos a los diferenciales de los bonos corporativos de alto rendimiento.

El vector tiene incluido a la tasa de bonos soberanos estadounidenses a 10 años, el componente cíclico del precio del petróleo, y se genera una variable dummie para los períodos de crisis, como la suscitada en 2008.

CUADRO 1. ESTADÍSTICA DESCRIPTIVA DE LAS VARIABLES

\begin{tabular}{|c|c|c|c|c|c|c|}
\hline & PERU & CHINA & TI & US & VIX & WTI \\
Mean & 4.947235 & 9.294 & 79.62862 & 2.540047 & 19.575 & 51.63386 \\
Median & 4.939246 & 8.9 & 79.01116 & 2.595905 & 17.455 & 48.14279 \\
Maximum & 13.46597 & 14.9 & 113.8545 & 5.29774 & 58.6 & 123.775 \\
Minimum & -5.330526 & 6.2 & 54.14936 & -3.92445 & 10.31 & 12.93365 \\
Std. Dev. & 3.439243 & 2.18377 & 19.81022 & 1.698022 & 7.496253 & 29.73508 \\
Skewness & -0.090564 & 0.62371 & 0.113463 & -1.421454 & 1.973054 & 0.498267 \\
Kurtosis & 3.439371 & 2.519284 & 1.427579 & 6.384486 & 9.703518 & 2.074219 \\
Jarque-Bera & 0.941059 & 7.44644 & 10.51668 & 81.40362 & 252.1205 & 7.708956 \\
Probability & 0.624671 & 0.024156 & 0.005204 & 0 & 0 & 0.021185 \\
Sum & 494.7235 & 929.4 & 7962.862 & 254.0047 & 1957.5 & 5163.386 \\
Sum Sq. Dev. & 1171.011 & 472.1164 & 38852.04 & 285.4446 & 5563.187 & 87533.33 \\
Observations & 100 & 100 & 100 & 100 & 100 & 100 \\
\hline
\end{tabular}


GRÁFICA 1. TENDENCIA DE LAS VARIABLES (SERIE DE TIEMPO)

PERU
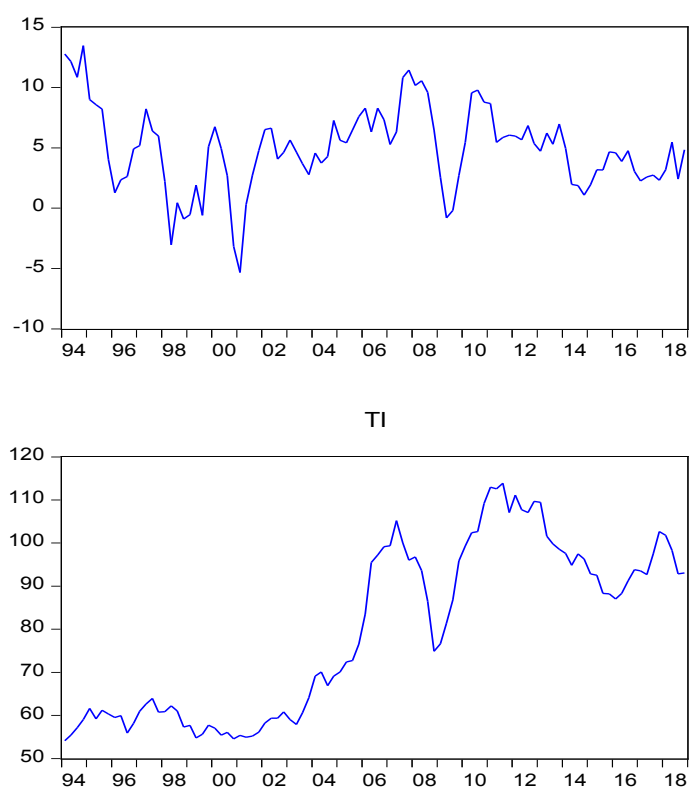

$\mathrm{VIX}$

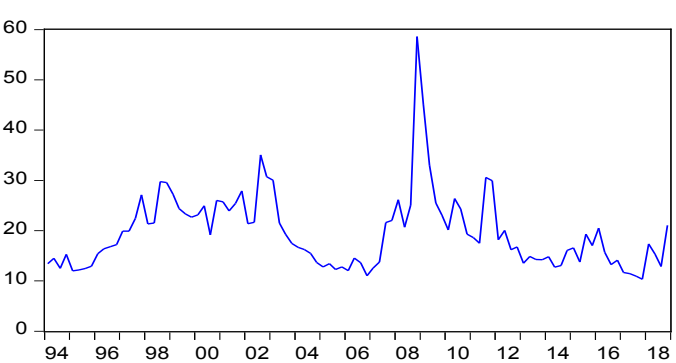

Antes de estimar el VARX descomponemos en su ciclo y tendencia a la variable wti usando el filtro Hodrick - Prescott (HP) (lambda=1,600), luego de ello nos quedamos con la parte cíclica (para incorporarla como variable exógena)

Analizando el VAR estimado se observa que es estable porque todas sus raíces inversas están dentro del círculo unitario. Para verificar si el

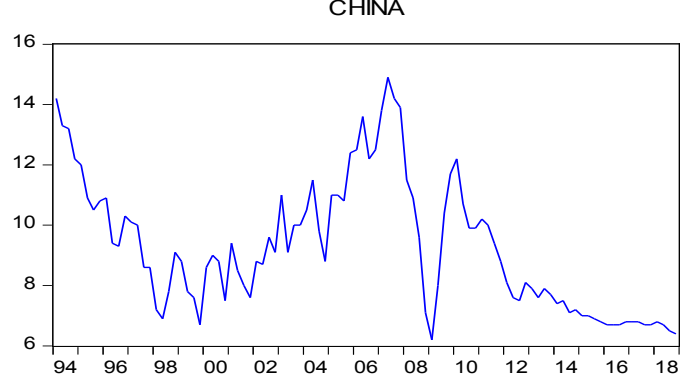

US
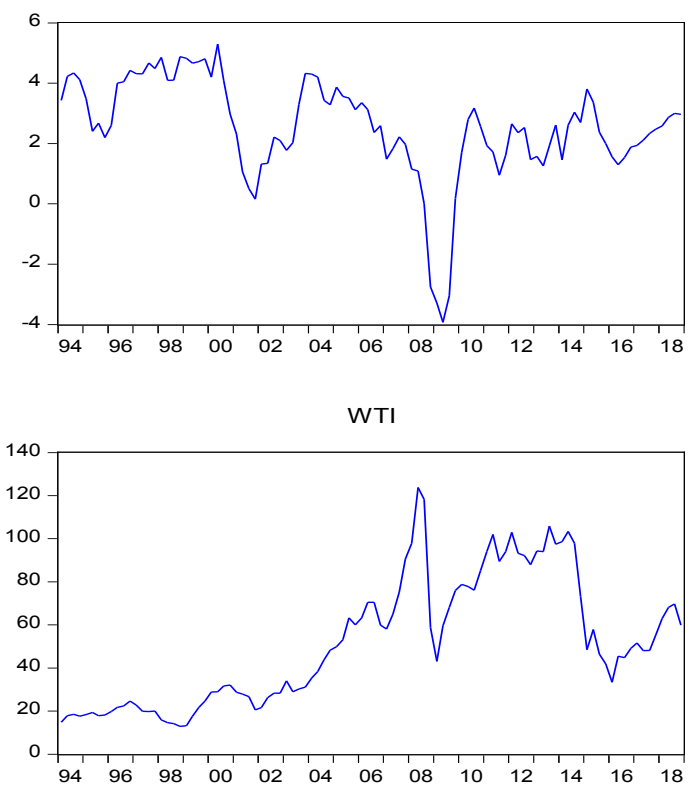

modelo está especificado correctamente se debe verificar que los residuos sean ruido blanco, entonces a través del software econométrico realizamos este análisis y se detecta que los residuos parecen ser ruido blanco (ver gráfica 2), aunque se obtienen algunos residuos grandes en ciertos períodos que parecen estar relacionados con los episodios de crisis (tienen información valiosa y es conveniente tenerlos en la muestra). 
GRÁFICA 2. ANÁLISIS DE RESIDUOS (VAR ESTIMADO)
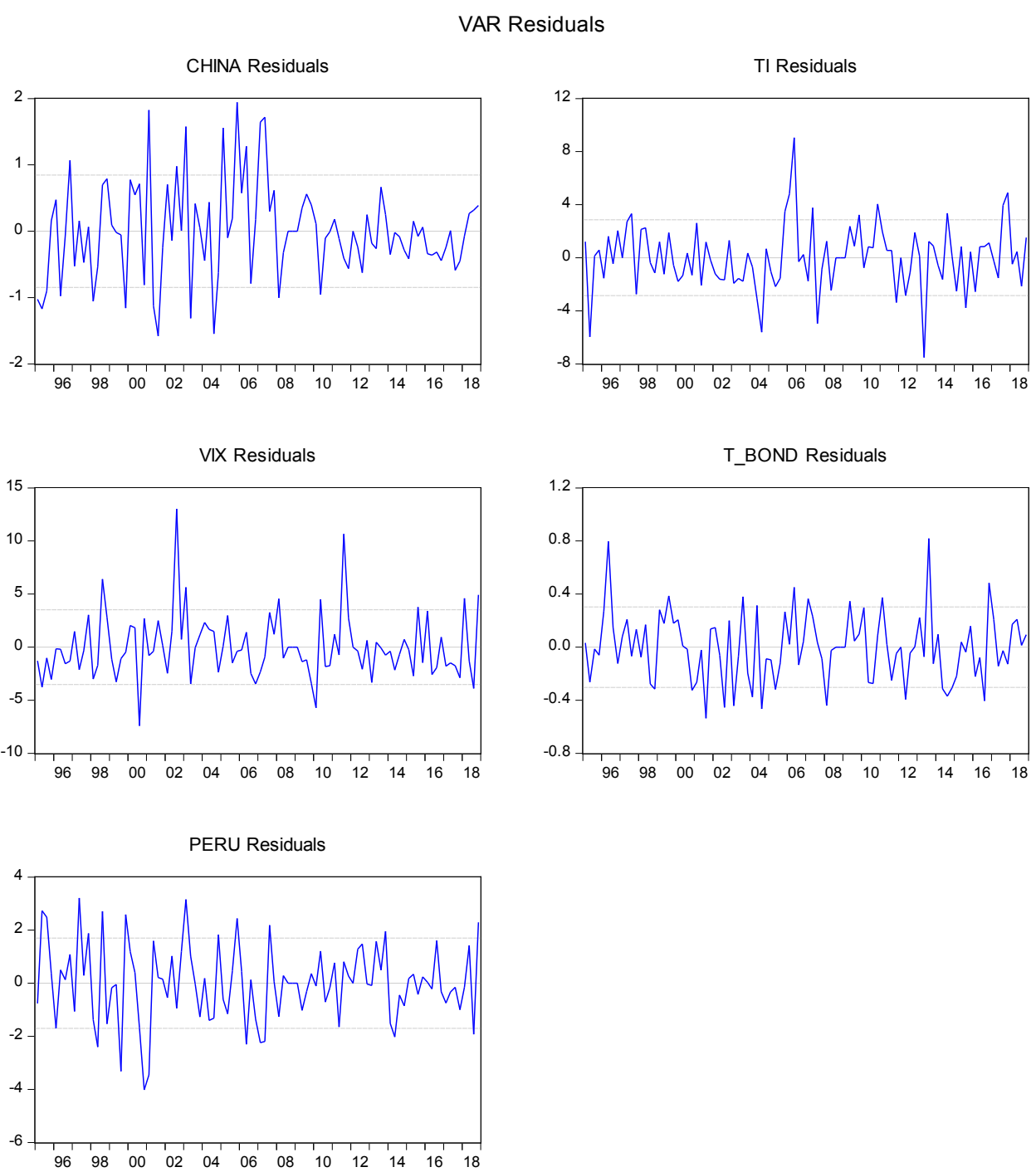

Aplicando la prueba de residuos, se obtienen los correlogramas cruzados, autocorrelaciones (con aproximadamente 2 límites de error estándar) para el VARX que se está estimando. Dado que los valores están dentro de las líneas punteadas en los gráficos (ver gráfica 3), se concluye que los residuos son aleatorios. 
GRÁFICA 3. PRUEBA DE RESIDUOS: CORRELOGRAMAS CRUZADOS (VAR ESTIMADO)

Autocorrelations with Approximate 2 Std. Err. Bounds

$\operatorname{Cor}($ CHINA,CHINA(-i))

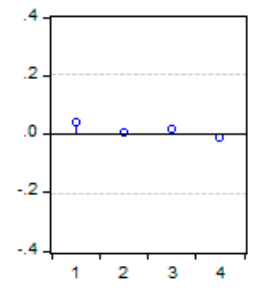

$\operatorname{Cor}(\mathrm{TI}, \mathrm{CHINA}(-\mathrm{i}))$

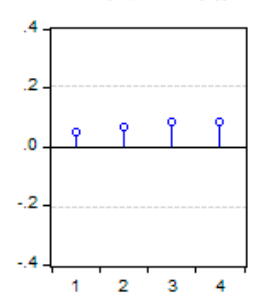

Cor(VIX,CHINA(-i))

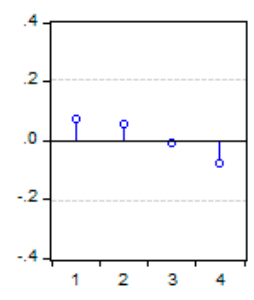

Cor(T_BOND,CHINA(-i))

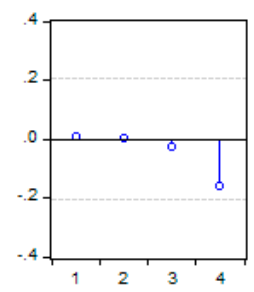

Cor(PERU,CHINA(-i))

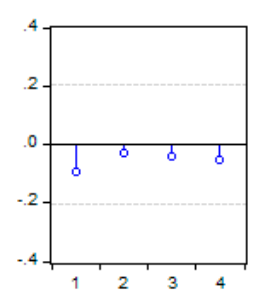

$\operatorname{Cor}(\mathrm{CHINA}, \mathrm{TI}(-\mathrm{i}))$

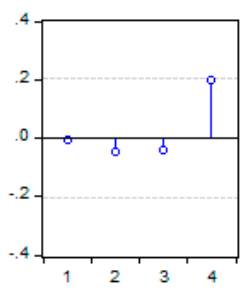

$\operatorname{Cor}(T I, T I(-1))$

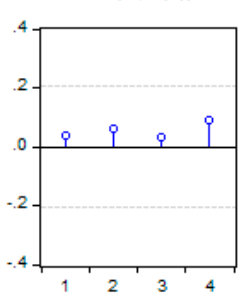

Cor(VIX,TI(-i))

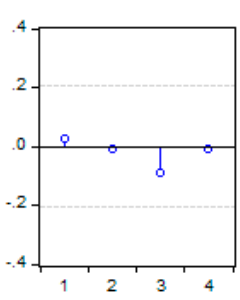

Cor(T_BOND,TI(-i))

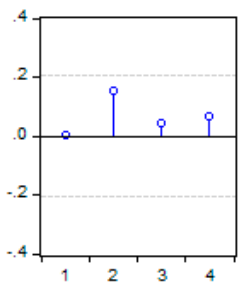

$\operatorname{Cor}($ PERU,TI(-i))

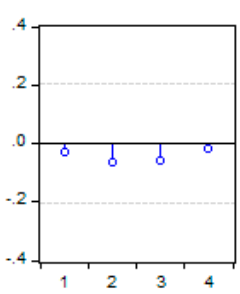

Cor(CHINA, VIX(-i))

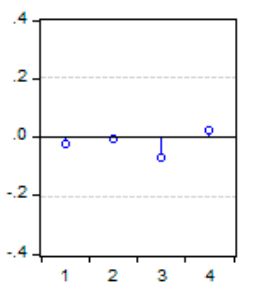

Cor(TI,VIX(-i))

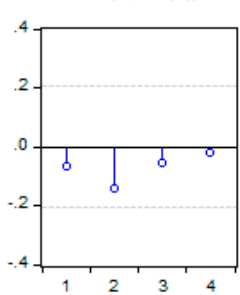

$\operatorname{Cor}(V I X, V I X(-i))$

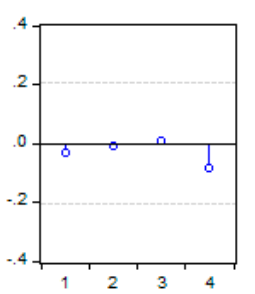

Cor(T_BOND,VIX(-i))

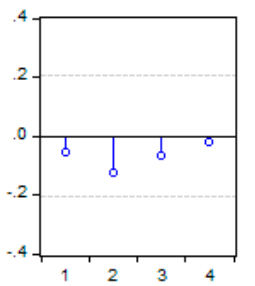

$\operatorname{Cor}($ PERU,VIX(-i))

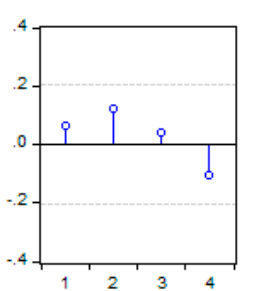

Cor(CHINA,T_BOND(-i))

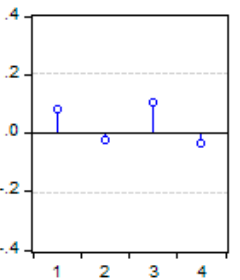

$\operatorname{Cor}(T 1, T+B O N D(-i))$

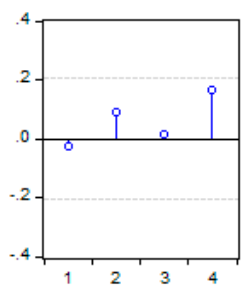

Cor(VIX,T_BOND(-i))

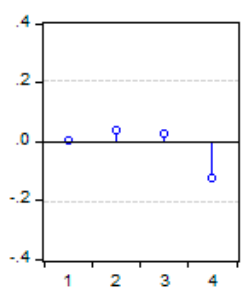

Cor(T_BOND,T_BOND(-i))

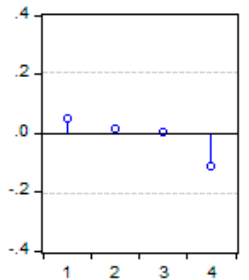

Cor(PERU,T_BOND(-i))

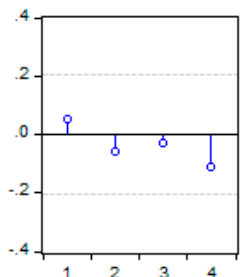

Cor(CHINA,PERU(-i))

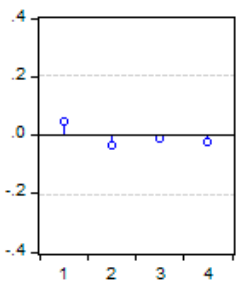

Cor(TI,PERU(-i))

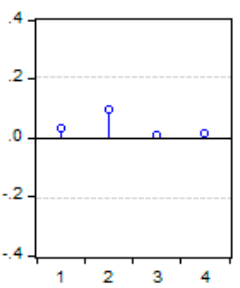

Cor(VIX,PERU(-i))

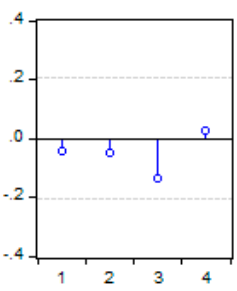

Cor(T_BOND,PERU(-i))

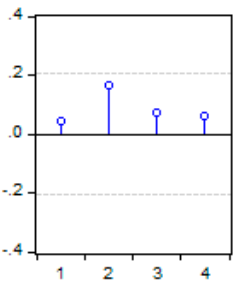

Cor(PERU,PERU(-i))

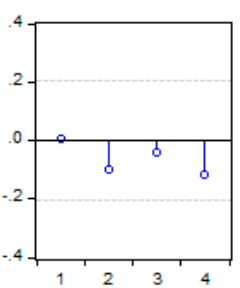




\section{RESULTADOS}

GRÁFICA 4. FUNCIONES IMPULSO-RESPUESTA DE LAS VARIABLES (VAR ESTIMADO)

Response to Cholesky One S.D. (d.f. adjusted) Innovations \pm 2 S.E.

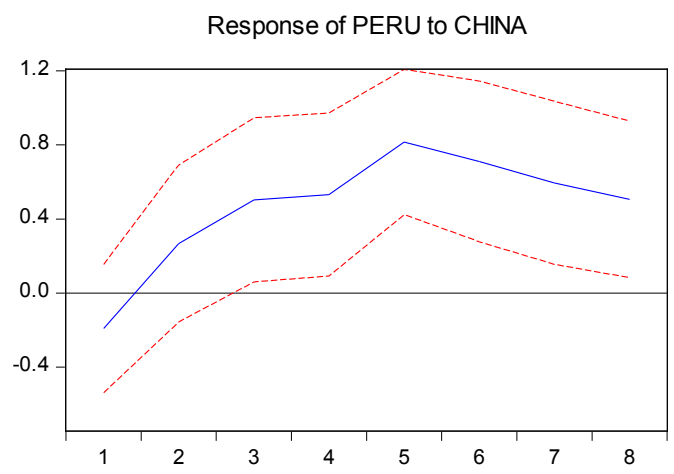

Response of PERU to TI

Response of PERU to VIX
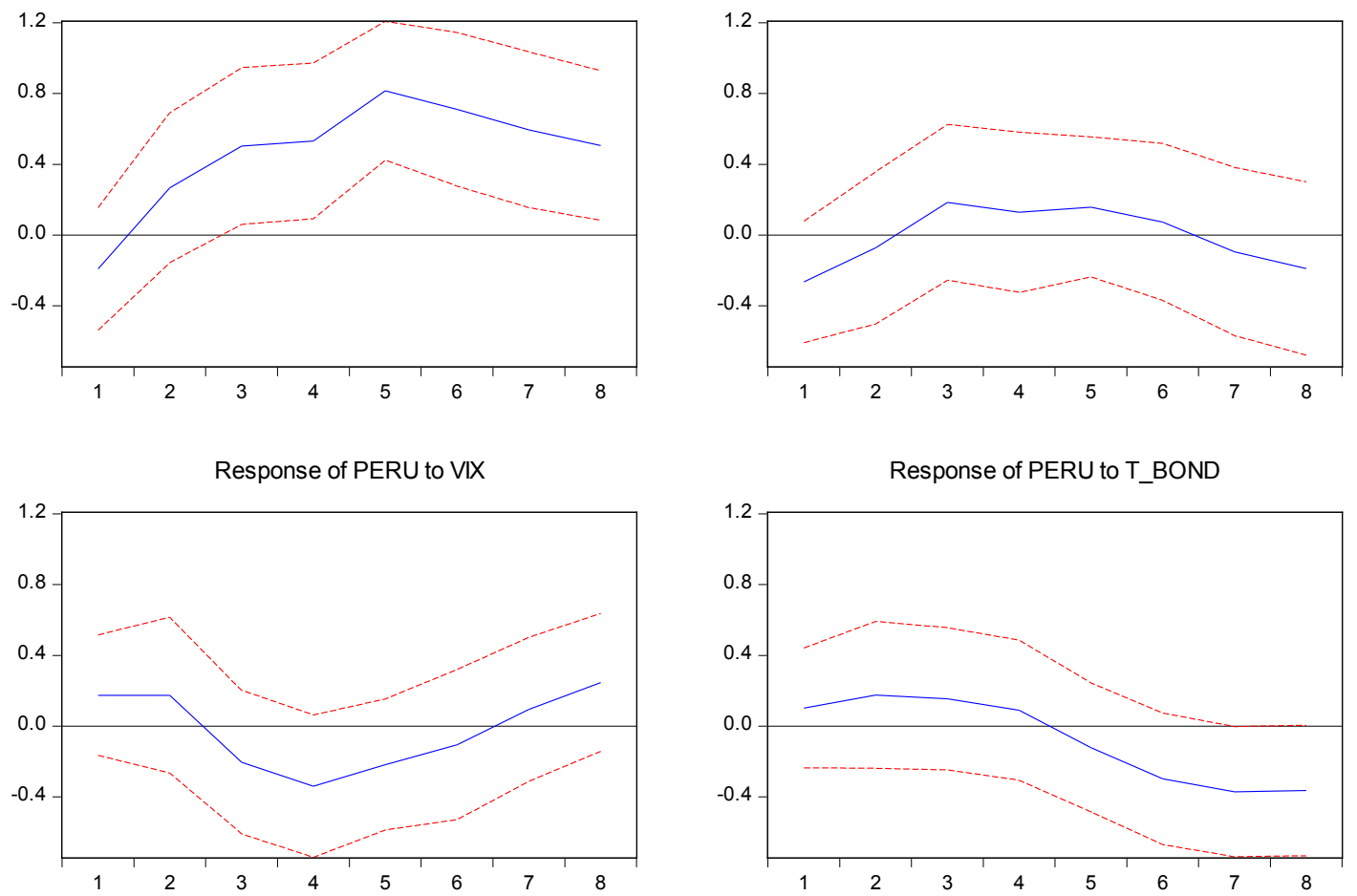

Response of PERU to T_BOND

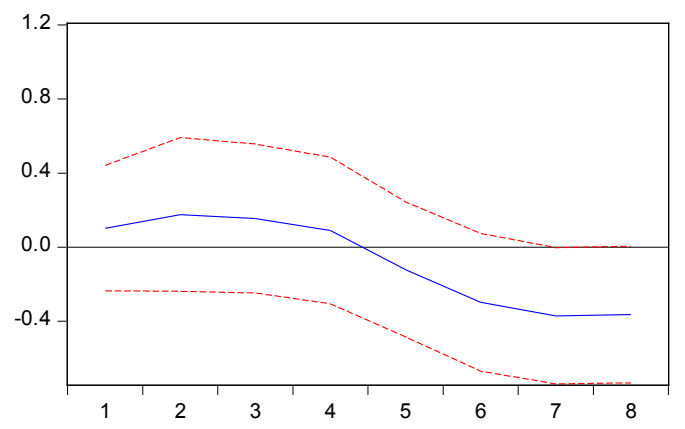

Se observa que la función impulso-respuesta de las variables analizadas con 8 períodos hacia adelante (ver gráfica 4), se puede observar que un aumento en el producto chino genera un incremento en el PBI nacional, y este impacto, a su vez, en el crecimiento, que dura aproximadamente dos años. Un impacto positivo en el VIX tiene un impacto negativo en el Producto, lo cual es de esperarse ya que esto es sinónimo de incertidumbre en los mercados financieros. 


\section{GRÁFICA 5. ACUMULADO DE LAS FUNCIONES IMPULSO-RESPUESTA DE LAS VARIA- BLES (VAR ESTIMADO)}

Accumulated Response to Cholesky One S.D. (d.f. adjusted) Innovations \pm 2 S.E.

Accumulated Response of PERU to CHINA

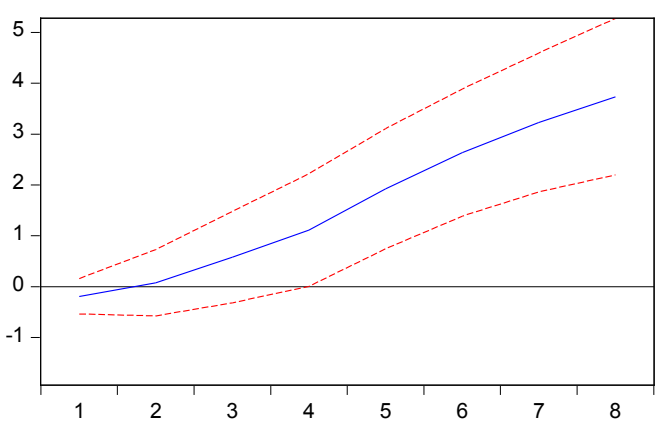

Accumulated Response of PERU to VIX

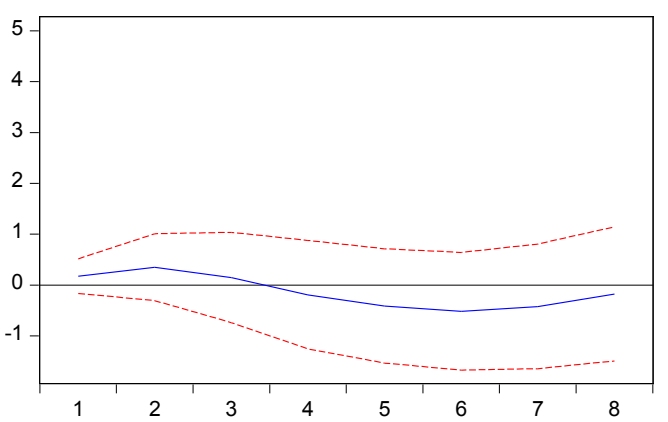

Accumulated Response of PERU to TI

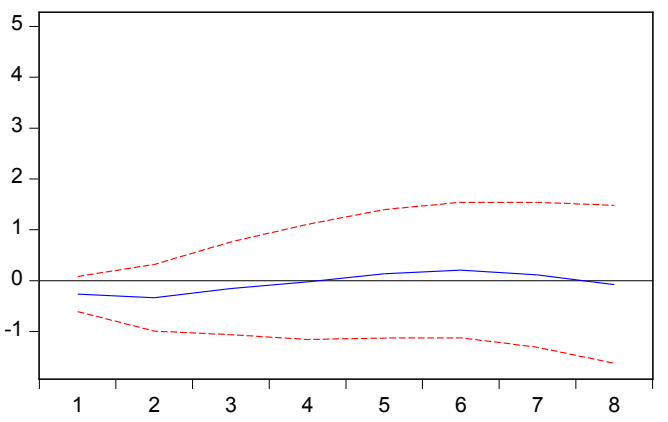

Accumulated Response of PERU to T_BOND

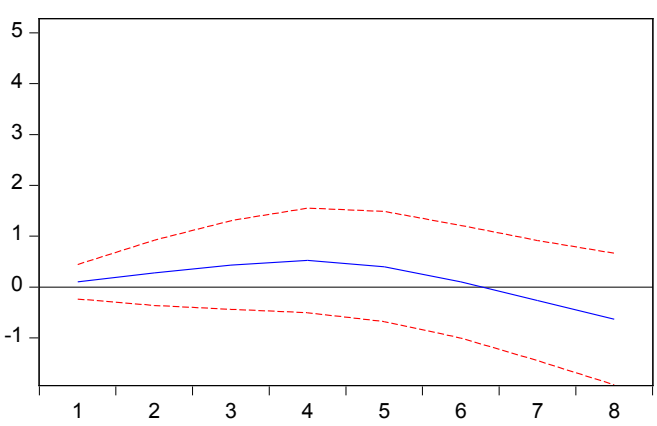

Se observa que las funciones de impulso-respuesta acumuladas (ver gráfica 5), guardan concordancia con lo descrito líneas atrás donde el gran impulsor del crecimiento del PBI peruano es el incremento del PBI de China. Como era de esperarse el índice de volatilidad financiera (VIX) y los aumentos en la tasa de los bonos del tesoro contribuyen de manera negativa en el PBI peruano.

Por otro lado, en el cuadro número 2 se observa la descomposición de la varianza, donde se desprende que al cabo de dos años el 30\% de la variabilidad del crecimiento del PBI peruano es explicado por la variabilidad del PBI de China, mientras que un 57\% es explicado por factores domésticos, lo que conlleva a decir que el $43 \%$ del crecimiento del PBI de Perú se explica por los factores externos.

\section{CUADRO 2. DESCOMPOSICIÓN DE LA VARIANZA DE LAS VARIABLES ANALIZADAS}

\begin{tabular}{rcccccc}
\hline \hline Period & S.E. & CHINA & TI & VIX & T BOND & PERU \\
\hline \hline 1 & 0.846008 & 1.263823 & 2.429009 & 1.054445 & 0.363060 & 94.88966 \\
2 & 1.074716 & 2.828935 & 1.976574 & 1.595642 & 1.086782 & 92.51207 \\
3 & 1.234930 & 8.487457 & 2.581683 & 2.410411 & 1.535253 & 84.98520 \\
4 & 1.308545 & 13.74027 & 2.698009 & 4.647301 & 1.566115 & 77.34831 \\
5 & 1.375009 & 22.64836 & 2.618753 & 4.587435 & 1.523993 & 68.62146 \\
6 & 1.461901 & 26.66678 & 2.306886 & 4.061858 & 2.598576 & 64.36590 \\
7 & 1.567508 & 29.12340 & 2.227312 & 3.831405 & 4.225064 & 60.59282 \\
8 & 1.651014 & 30.48839 & 2.538404 & 4.354635 & 5.624222 & 56.99435 \\
\hline \hline
\end{tabular}

Cholesky Ordering: CHINA TI VIX T BOND PERU 


\section{CONCLUSIONES Y RECOMENDACIONES}

Después de los análisis realizados, se pone en evidencia que Perú es sensible a los choques externos. Los shocks externos, tanto real como financiero, tienen un impacto significativo en el PBI peruano y podría decirse que son estos factores los que causan las fluctuaciones de ciclo económico de Perú en el período analizado. Como se mostró anteriormente, un impacto positivo en la producción de China suele ser expansivo en nuestra economía.

Ahora dado el gran impacto potencial de un fuerte deterioro del entorno externo, a raíz de la agudización de las fricciones comerciales, Perú debe mantener solidez en sus fundamentos macroeconómicos tanto fiscales como monetarios, para fortalecer la posición externa de Perú.

\section{REFERENCIAS}

[1] Abrego, L., \& Österholm, P. (2010). External linkages and economic growth in Colombia: Insights from a Bayesian VAR model. The World Economy, 1788-1810.

[2] Adler, G., \& Sosa, S. (2011). Commodity price cycles: The perils of mismanaging the boom. International Monetary Fund. IMF Working Paper.

[3] Bloom, N. (2009). The impact of uncertainty shocks. Econometrica, 623-685.

[4] Calvo, G. A., \& Talvi, E. (2005). Sudden stop, financial factors and economic collpase in Latin America. National Bureau of Economic Research.

[5] Calvo, G. A., Leiderman, L., \& Reinhart, C. M. (1993). Capital inflows and real exchange rate appreciation in Latin America: the role of external factors. International Monetary Fund. IMF Working Papers, 108-151.

[6] Castillo, P., Montoro, C., \& Tuesta, V. (2007). Hechos estilizados de la economía peruana. Estudios Económicos, 33-75.

[7] Chinn, M. D., \& Ito, H. (2006). What matters for financial development? Capital controls, institutions, and interactions. Journal of development economics, 163192.
[8] Corbo, V., \& Schmidt-Hebbel, K. (2011). The international crisis and Latin America: Growth effects and development strategies. CASE Network Studies \& Analyses.

[9] Izquierdo, A., Romero-Aguilar, R., \& Talvi, E. (2008). Booms and busts in Latin America: the role of external factors.

[10] Lütkepohl, H. (2007). General-to-specific or specific-to-general modelling? An opinion on current econometric terminology. Journal of Econometrics, 319-324.

[11] Roch, M. F. (2017). The adjustment to commodity price shocks in Chile, Colombia, and Peru. International Monetary Fund. IMF Working Paper.

[12] Saldarriaga, M. Á., \& Winkelried, D. (2013). Trade linkages and growth in Latin America: An SVAR analysis. International Economics, 13-28.

[13] Sims, C. A. (1980). Macroeconomics and reality. Econometrica: journal of the Econometric Society, 1-48.

[14] Sosa, S. (2012). Output Fluctuations in Chile-The Role of External Factors. INTERNATIONAL MONETARY FUND. IMF Working Papers.

[15] Stock, J. H., \& Watson, M. W. (2001). Vector Autoregressions. Journal of Economic Perspectives, 101-115. doi:10.1257/ jep.15.4.101

[16] Tello, M. D. (2012). Peru: integration, sectoral specialization and synchronization with international gross domestic product cycles. CEPAL.

[17] Zettelmeyer, J., \& Österholm, P. (2007). The Effect of External Conditionson Growth in Latin America. International Monetary Fund. IMF Working Paper. 